\title{
THE EQUTVALENCE OF $\times{ }^{t} C \approx \times^{t} D$ AND $J \times C \approx J \times D$ \\ BY \\ RONALD HIRSHON
}

\begin{abstract}
Let $C$ satisfy the maximal condition for normal subgroups and let $X^{\prime} C \approx X^{\prime} D$ for some positive integer $t$. Then $C \times J \approx D \times J$ where $J$ is the infinite cyclic group. If $X^{s} C \approx X^{t} D$ and $s>t$, there exists a finitely generated free abelian group $S$ such that $C$ is a direct factor of $D \times S$.
\end{abstract}

1. Introduction. Let $M$ be a group and let

$$
M \times C \approx M \times D .
$$

Let $R$ be the group such that (1) always implies that $R \times C \approx R \times D$. We say then that $M$ is $R$ replaceable. In particular, if $R$ is the identity group, a group $M$ is $R$ replaceable if and only if $M$ is cancelable in direct products. Our main interest in this concept is where $R=1$, or $R=J$, the infinite cyclic group. We have recently shown the following result [3]:

THEOREM A. Let $E / E^{\prime \prime}$ be finitely generated, and let $E / Z(E)$ be hopfian, where $Z(E)$ is the center of $E$. Furthermore, let $E / Z(E)$ obey the minimal condition on direct factors. Then $E$ is $J$ replaceable.

Note the above result implies that if $E$ satisfies the maximal condition on normal subgroups then $E$ is $J$ replaceable.

If (1) always implies that there exists a positive integer $t$ such that the Cartesian direct product of $t$ copies of $C, \times{ }^{t} C$, satisfies $\times^{t} C \approx \times^{t} D$ then we say that $M$ is power cancelable. $J$ is power cancelable [4]. Consequently any $J$ replaceable group is power cancelable.

In $\$ 2$ we show a partial converse to the result that $J$ is power cancelable. We show that if $C$ satisfies the maximal condition on normal subgroups and there exists a positive integer $t$ with $\times^{t} C \approx \times^{t} D$, then $C \times J \approx D \times J$. We also study the relation $\times^{s} C \approx \times^{t} D, s \neq t$. If $s \geqslant t$, we show that there exists a finitely generated free abelian group $S$ such that $C$ is a direct factor of $D \times S$.

The above results depend heavily on the fact that groups with the maximal condition for normal subgroups are $J$ replaceable. It seems natural to wonder about how far the concept of $J$ replacement can be extended.

Received by the editors July 11, 1977.

AMS (MOS) subject classifications (1970). Primary 20F30; Secondary 20F15.

$K e y$ words and phrases. $J$ replacement, cancelation. 
We note that there exists a finitely generated group $F, F \neq 1$, with $F \approx F \times F[8]$. Hence finitely generated groups are not in general $J$ replaceable. The main object of $\S 3$ is to obtain sufficient conditions for a finitely generated group to be $J$ replaceable. We do this in terms of the normal subgroup structure of the group. Our methods simultaneously yield sufficient conditions for the cancelability of certain groups in direct products. Some samples of our results in this section are the following:

THEOREM. Let $E$ be a finitely generated group with a hopfian commutator subgroup $E^{\prime}$. Suppose $E^{\prime}$ decomposes into a free product. Then $E$ is $J$ replaceable.

Let $G(n, m)$ be the group on two generators $a, b$ with single defining relation $a^{n}=b^{m}$. Direct products of such groups do not behave pleasantly with respect to uniqueness of decompositions into directly indecomposable factors. For example $G(2,2) \times G(3,3)$ has a decomposition in which $J$ is a direct factor [9]. However the commutator subgroup of $G(n, m)$ is a finitely generated free group. Hence we may deduce the

COROLlaRY. $G(n, m)$ is $J$ replaceable..

\section{Taking roots in direct products.}

2.1 Some preliminary arguments.

Lemma 1. Let $G=A \times B=C \times D$. Then

and

$$
R=\left[\left(A / A^{\prime}\right) \times C \times D\right] \approx\left[C /\left(A^{\prime} \cap C\right)\right] \times\left[D /\left(A^{\prime} \cap D\right)\right] \times A
$$

$$
S=\left[\left(C / C^{\prime}\right) \times A \times B\right] \approx\left[A /\left(A^{\prime} \cap C\right)\right] \times\left[B /\left(B^{\prime} \cap C\right)\right] \times C .
$$

Proof. Since $A^{\prime}=\left(A^{\prime} \cap C\right) \times\left(A^{\prime} \cap D\right)$ we have $\left(G / A^{\prime}\right) \approx\left(A / A^{\prime}\right) \times B$ $\approx\left(C / A^{\prime} \cap C\right) \times\left(D / A^{\prime} \cap D\right)$. Hence $\left(A / A^{\prime}\right) \times B \times A \approx\left(C / A^{\prime} \cap C\right) \times$ $\left(D / A^{\prime} \cap D\right) \times A$ and the first assertion of the lemma follows by replacing $B \times A$ by $C \times D$. The second assertion follows symmetrically.

LEMMA 2. If $P$ and $Q$ are any groups with $P \times J \approx Q \times J \times J$, then $P \approx Q \times J$.

Proof. See Lemma 1 of [5].

LEMMA 3. Let $G=A \times B=C \times D$ and let $\left(A / A^{\prime}\right) \approx\left(C / C^{\prime}\right)$. Furthermore suppose $B$ and $A /\left(A^{\prime} \cap C\right)$ are $J$ replaceable. Then $\left[D /\left(A^{\prime} \cap D\right)\right] \times J$ $\approx\left[B /\left(C \cap B^{\prime}\right)\right] \times J$.

$$
\begin{aligned}
& \text { Proof. } G /\left(A^{\prime} \cap C\right) \approx\left(A / A^{\prime} \cap C\right) \times B \approx\left(C / A^{\prime} \cap C\right) \times D \text { so that } \\
& \qquad \begin{array}{r}
\left(A / A^{\prime} \cap C\right) \times B \times\left(D / A^{\prime} \cap D\right) \\
\approx\left[\left(C / A^{\prime} \cap C\right) \times\left(D / A^{\prime} \cap D\right)\right] \times D .
\end{array}
\end{aligned}
$$


Now the term in brackets in (2) is isomorphic to $\left(G / A^{\prime}\right)$. However $G / A^{\prime} \approx$ $\left(A / A^{\prime}\right) \times B$ so that

$$
\left(A / A^{\prime} \cap C\right) \times B \times\left(D / A^{\prime} \cap D\right) \approx\left(A / A^{\prime}\right) \times B \times D,
$$

so that replacing $B$ by $J$ above, we see

$$
\left(A / A^{\prime} \cap C\right) \times\left(D / A^{\prime} \cap D\right) \times J \approx\left(A / A^{\prime}\right) \times D \times J .
$$

Hence

$$
\left(A / A^{\prime} \cap C\right) \times\left(D / A^{\prime} \cap D\right) \times J \approx\left(C / C^{\prime}\right) \times D \times J .
$$

Since

we have

$$
\begin{aligned}
\left(C / C^{\prime}\right) \times D & \approx(C \times D) / C^{\prime}=(A \times B) / C^{\prime} \\
& \approx\left(A / C \cap A^{\prime}\right) \times\left(B / C \cap B^{\prime}\right),
\end{aligned}
$$

$$
\begin{aligned}
\left(A / A^{\prime} \cap C\right) \times\left(D / A^{\prime} \cap D\right) \times J & \\
& \approx\left(A / C \cap A^{\prime}\right) \times\left(B / C \cap B^{\prime}\right) \times J .
\end{aligned}
$$

We may now replace $\left(A / A^{\prime} \cap C\right)$ by $J$ in both sides of (3) and then by Lemma 2 cancel one factor $J$ from both sides of the resulting isomorphism.

2.2 The main result.

Theorem 1. If $\times{ }^{n} F \approx \times{ }^{n} G$ and $F$ satisfies the maximal condition for normal subgroups then $F \times J \approx G \times J$.

Proof. Let $F_{1} \times F_{2} \times \cdots \times F_{n}=G_{1} \times G_{2} \times \cdots \times G_{n}$ where $F_{i} \approx F$, $G_{i} \approx G, 1 \leqslant i \leqslant n$, and $F$ satisfies the maximal condition on normal subgroups. We proceed indirectly. Suppose $F \times J$ and $G \times J$ are not isomorphic. Consider the pairs of groups $F_{L}, L$ such that

$$
\times{ }^{n}\left[\left(F / F_{L}\right) \times J\right] \approx \times^{n}(L \times J), \quad\left(F / F_{L}\right) \times J \approx L \times J .
$$

One such pair is given by the pair $1, G$. Choose a pair $F_{M}, M$ with $F_{M}$ maximal in $F$. Let $H=\left(F / F_{M}\right) \times J, E=M \times J$. Let $H_{1} \times H_{2}$ $\times \cdots \times H_{n}=E_{1} \times E_{2} \times \cdots \times E_{n}$ with $H_{i} \approx H, E_{1} \approx E, 1<i<n$. Now since $H_{1}^{\prime}=\times_{i=1}^{n}\left(H_{1} \cap E_{i}^{\prime}\right)$ if $H_{1} \cap E_{i}^{\prime}=1$ for all $i, H_{1}$ is abelian and hence finitely generated. This would imply $H \approx E$, contrary to assumption. Hence we may suppose that $H_{1} \cap E_{1}^{\prime} \neq 1$. Now let $A=H_{1}, B=H_{2} \times H_{3}$ $\times \cdots \times H_{n}, C=E_{1}, D=E_{2} \times E_{3} \times \cdots \times E_{n}$ so that $A \times B=C \times D$. From $\times^{n}\left(A / A^{\prime}\right) \approx \times^{n}\left(C / C^{\prime}\right)$ we deduce $\left(A / A^{\prime}\right) \approx\left(C / C^{\prime}\right)$ so that the groups $R$ and $S$ of Lemma 1 are isomorphic so that $\times^{n} R \approx \times{ }^{n} S$. We may write $\left(X^{n} R\right) \times\left(X^{n} J\right) \approx\left(X^{n} S\right) \times\left(X^{n} J\right)$ as

$$
\begin{aligned}
\times{ }^{n}\left(C / A^{\prime} \cap C\right) & \times\left(\times^{n} D_{*}\right) \times\left(\times^{n} A\right) \\
& \approx \times^{n}\left(A / A^{\prime} \cap C\right) \times\left(\times^{n} B_{*}\right) \times\left(\times^{n} C\right)
\end{aligned}
$$


where $D_{*} \approx\left[D /\left(A^{\prime} \cap D\right)\right] \times J$ and $B_{*} \approx\left[B /\left(B^{\prime} \cap C\right)\right] \times J$. By Lemma 3, $D_{*} \approx B_{*}$. Hence we conclude by $J$ replacement that $\left[\times^{n}\left(C / A^{\prime} \cap C\right)\right] \times J$ $\approx\left[\times^{n}\left(A / A^{\prime} \cap C\right)\right] \times J$. Hence if $C_{*}=\left[C /\left(A^{\prime} \cap C\right)\right] \times J, A_{*}=$ $\left[A /\left(A^{\prime} \cap C\right)\right] \times J$, then $\times{ }^{n} C_{*} \approx \times{ }^{n} A_{*}$. Since $A^{\prime} \cap C \neq 1$, we conclude from the maximality of $F_{M}$ that $C_{*} \approx A_{*}$. Hence the isomorphism $R \times J \times$ $J \approx S \times J \times J$ may be rewritten as $C_{*} \times D_{*} \times A \approx A_{*} \times B_{*} \times C$, so that by $J$ replacement $A \times J \approx C \times J$, contrary to assumption.

COROLlary. If $F$ is finitely generated and $\times^{n} F \approx \times{ }^{n} G$ then $\left(F / F^{\prime \prime}\right) \times J$ $\approx\left(G / G^{\prime \prime}\right) \times J$.

Proof. $X^{n}\left(F / F^{\prime \prime}\right) \approx \times^{n}\left(G / G^{\prime \prime}\right)$ and $F / F^{\prime \prime}$ satisfies the maximal condition on normal subgroups [2].

$2.3 \times^{s} A \approx X^{r} B$.

Theorem 1 naturally leads us to wonder about what may be said about decompositions of the form $X^{s} A \approx X^{r} B, s \neq r$. In this direction we have

TheOREM 2. Let $X^{s} A=X^{r} B$ with $s \geqslant r \geqslant 2$ and where $A$ satisfies the maximal condition for normal subgroups. Then there exists a finitely generated free abelian group $S$ such that $A$ is a direct factor of $B \times S$.

\subsection{Some preliminary arguments.}

Lemma 4. Let $A_{1} \times A_{2}=B_{1} \times B_{2}=G$ and let $F$ be a normal subgroup of $G, F \subset A_{1} \cap B_{1}$. Let $B_{2}$ or $A_{2}$ be $J$ replaceable. Then $\left(A_{1} / F\right) \times B_{1} \times J \approx A_{1}$ $\times\left(B_{1} / F\right) \times J$.

Proof. $G / F \approx\left(A_{1} / F\right) \times A_{2} \approx\left(B_{1} / F\right) \times B_{2}$. Hence $\left(A_{1} / F\right) \times A_{1} \times A_{2}$ $\approx\left(B_{1} / F\right) \times A_{1} \times B_{2}$ and so $\left(A_{1} / F\right) \times B_{1} \times B_{2} \approx\left(B_{1} / F\right) \times A_{1} \times B_{2}$. If $B_{2}$ is $J$ replaceable we may now replace $B_{2}$ by $J$ in the above isomorphism.

Lemma 5. Let $G=M \times C_{*}=L \times E_{*}$ where $C_{*}=C_{1} \times C_{2} \times \cdots \times C_{k}$ and $E_{*}=E_{1} \times E_{2} \times \cdots \times E_{k}$ and where $E_{i} \approx E_{j}=E, C_{i} \approx C_{j} \approx C, i \neq j$, and $C$ and $M$ are $J$ replaceable. Let $F$ be a normal subgroup of $G, F \subset E_{1} \cap$ $C_{1}$. Then $J \times M \times\left[\times^{k}\left(C_{1} / F\right)\right] \approx J \times L \times\left[\times^{k}\left(E_{1} / F\right)\right]$.

PRoof. By using the previous lemma we see

$$
\begin{aligned}
\left(\times^{k} J\right) \times\left[\times^{k}\left(E_{1} / F\right)\right] \times C_{*} \times L & \approx \times^{k}\left[\left(E_{1} / F\right) \times C_{1} \times J\right] \times L \\
& \approx \times^{k}\left[E_{1} \times\left(C_{1} / F\right) \times J\right] \times L \\
& \approx\left[\times^{k}\left(C_{1} / F\right)\right] \times E_{*} \times L \times\left(\times^{k} J\right) \\
& \approx\left[\times^{k}\left(C_{1} / F\right)\right] \times M \times C_{*} \times\left(\times^{k} J\right) .
\end{aligned}
$$

If we now focus our attention on the first and last terms in the above 
isomorphisms, we may replace $C_{*}$ by $J$ in the corresponding expressions and then invoke Lemma 2 to obtain the desired result.

2.5 Proof of Theorem 2.

Consider the pairs of groups $K, A_{K}$ such that

$$
J \times\left(\times^{s-r} A\right) \times\left[\times^{r}\left(A / A_{K}\right)\right] \approx J \times\left(\times^{r} K\right) .
$$

One such pair is given by $K=B, A_{B}=1$. Choose a pair $N, A_{N}$ with $A_{N}$ maximal in $A$. Hence we may write

$$
M \times C_{1} \times C_{2} \times \cdots \times C_{r}=J \times E_{1} \times E_{2} \times \cdots \times E_{r}
$$

with $M \approx\left(X^{s-r} A\right) \times J, C_{i} \approx\left(A / A_{N}\right), E_{i} \approx N, 1 \leqslant i \leqslant r$. Now apply Lemma 5 with $F=C_{1} \cap E_{1}$ and then replace $J \times J$ by $J$ in both sides of the resulting isomorphism. We obtain

$$
J \times\left(\times^{s-r} A\right) \times\left[\times^{r}\left(C_{1} / F\right)\right] \approx J \times\left[\times^{r}\left(E_{1} / F\right)\right] .
$$

By the maximality of $A_{N}, F=1$. That is $C_{1} \cap E_{1}=1$. Similarly $C_{i} \cap E_{j}=1$, $1<j<r, 1<i<r$. Hence $C_{1}$ is a finitely generated abelian group. If $C_{1} \approx T \times F$ with $T$ torsion free and $F$ finite then $N$ must have a direct factor isomorphic to $F$ so that we may write $N \approx F \times D$ for some $D$. Hence from (5)

$$
J \times\left(X^{s-r} A\right) \times\left(X^{r} T\right) \approx J \times\left(X^{r} D\right)
$$

Hence

$$
\left(X^{s} J\right) \times\left[\times^{(s-r) s} A\right] \times\left(X^{s r} T\right) \approx\left(X^{s} J\right) \times\left(X^{s r} D\right)
$$

so that

$$
\left(X^{s} J\right) \times\left[X^{(s-r) r} B\right] \times\left(X^{s r} T\right) \approx\left(X^{s} J\right) \times\left(X^{s r} D\right) .
$$

Hence from Lemma 2 we may replace $X^{s} J$ by $X^{r} J$ on both sides of the above isomorphism and then apply Theorem 2 (by taking $r$ th roots). We obtain (after canceling a superfluous factor of $J$ from both sides)

$$
J \times\left(X^{s-r} B\right) \times\left(X^{s} T\right) \approx J \times\left(\times^{s} D\right) .
$$

If we write $X^{s} D \approx\left(X^{s-r} D\right) \times\left(X^{r} D\right)$, we see from (6) and (7) that

$$
J \times\left(X^{s-r} A\right) \times\left(X^{r} T\right) \times\left(X^{s-r} D\right) \approx J \times\left(X^{s-r} B\right) \times\left(X^{s} T\right) .
$$

By using Lemma 2 this reduces to

$$
J \times\left(X^{s-r} A\right) \times\left(X^{s-r} D\right) \approx J \times\left(X^{s-r} B\right) \times\left(X^{s-r} T\right) .
$$

Now replace the first factor $J$ on each side of the isomorphism above by $X^{s-r J}$. We may then apply Theorem 2 and take an $(s-r)$ th root. After canceling a superfluous factor of $J$ we obtain

$$
J \times A \times D \approx B \times J \times T
$$

so that we may take $S=J \times T$. 


\section{Some $J$ replacement results.}

3.1 Preliminary concepts.

Definition 1. We say that the subgroup $M$ of a group $E$ is a prefactor of $E$ if $M$ is a homomorphic image of $E$ and $E$ is generated by $M$ and the centralizer of $M$ in $E$. We say that $M$ is a proper prefactor of $E$ if $M$ is a prefactor of $E$, a proper subgroup of $E$, and a proper homomorphic image of $E$. That is, $M \approx E / E_{M}, E_{M} \neq 1$ for some $E_{M}$.

Definition 2. We say that a group $E$ satisfies the minimal condition on proper prefactors if every descending chain $E=E_{1} \supset E_{2} \supset E_{3} \supset \ldots$ with $E_{i+1}$ a proper prefactor of $E_{i}$ must be a finite chain.

Definition 3. We say that $E$ is a HIR group if $E^{\prime}$ does not contain two infinite sequences of normal subgroups of $E$,

$$
K_{1}, K_{2}, K_{3}, \ldots, K_{1} \neq 1, \quad K_{i} \approx K_{j}, \quad 1 \leqslant i \leqslant j<\infty
$$

and

$$
L_{1}, L_{2}, L_{3}, \ldots, L_{i} \approx L_{j}, \quad 1 \leqslant i \leqslant j<\infty,
$$

and a prefactor $R$ of $E, L_{1} \approx R^{\prime}$, such that for any positive integer $n$,

$$
R^{\prime}=L_{n} \times K_{1} \times K_{2} \times K_{3} \times \cdots \times K_{n} \text {. }
$$

(HIR, for lack of a better name, stands for "Helpful In Replacement".)

Clearly if $E$ satisfies either the maximal condition for normal subgroups or the minimal condition for normal subgroups then $E$ is an HIR group and satisfies the minimal condition on proper prefactors. If $E$ is finitely generated and $E^{\prime}$ satisfies the minimal condition on prefactors and has hopfian normal prefactors then $E$ satisfies the minimal condition on proper prefactors. To see the above let $E=E_{1} \supset E_{2} \supset E_{3} \supset \ldots$ be a descending chain, $E_{i+1}$ a prefactor of $E_{i}$. Let $\theta_{i}$ be a homomorphism of $E_{i}$ onto $E_{i+1}$. Then $\theta_{i}$ induces a homomorphism $\bar{\theta}_{i}$ of $E_{i} / E_{i}^{\prime}$ onto $E_{i+1} / E_{i+1}^{\prime}$, and if $\alpha_{n}$ is the composite of $\bar{\theta}_{1}, \bar{\theta}_{2}, \ldots, \bar{\theta}_{n}, \alpha_{n}$ induces a homomorphism of $E / E^{\prime}$ onto $E_{n} / E_{n}^{\prime}$. If $K_{n}$ is the kernel of $\alpha_{n}$, ultimately, since $E / E^{\prime}$ satisfies the maximal condition on normal subgroups, $K_{n}=K_{n+1}$. This implies $\bar{\theta}_{n+1}$ is an isomorphism. Hence, ultimately, the kernel of $\theta_{n}$ is contained in $E_{n}^{\prime}$. However, $E_{1}^{\prime} \supset E_{2}^{\prime} \supset E_{3}^{\prime}$ $\supset \ldots$ is a descending chain of prefactors of $E^{\prime}$ so that ultimately all the $E_{i}^{\prime}$ are identical, say $E_{i}^{\prime}=E_{i+1}^{\prime}, i \geqslant p$. Hence, ultimately, $\theta_{i}$ induces a surjective endomorphism of the hopfian group $E_{i}^{\prime}$ so that ultimately $\theta_{i}$ is an isomorphism.

We may also note that a group with a single defining relation is an HIR group. For if a group fails to be an HIR group it contains a sequence of mutually distinct nontrivial subgroups $K_{i}, i>1$, such that the $K_{i}$ generate a direct product $K=K_{1} \times K_{2} \times \ldots$ But then if we choose $k_{i} \in K_{i}, k_{i} \neq 1$, we see that the $k_{i}$ generate an abelian subgroup which is neither cyclic, free abelian of rank 2 or torsion free of rank 1, contrary to [10]. 


\subsection{Statement of results.}

THEOREM 3. Let $E$ obey the minimal condition on proper prefactors and be an HIR group. Let $R$ be a group such that $R \times R$ is $R$ replaceable and such that the abelian homomorphic images of $E$ are $R$ replaceable. Then $E$ is $R$ replaceable.

The following are immediate consequences of Theorem 3:

COROLlARY 1. If $E$ is a finitely generated HIR group obeying the minimal condition on proper prefactors then $E$ is $J$ replaceable.

COROLLARY 2. If $E$ is a finitely generated group such that

(a) $E^{\prime}$ satisfies the minimal condition on prefactors and

(b) $E^{\prime}$ has hopfian prefactors, then $E$ is $J$ replaceable.

Also since a nontrivial free product cannot have a proper prefactor (see the proof of Theorem 6 of [5]), we see

COROLlaRY 3. Let $E$ be a finitely generated group such that $E^{\prime}$ is a free product, $E^{\prime}=E_{1} * E_{2}, E_{1} \neq 1, E_{2} \neq 1$. Furthermore suppose that $E^{\prime}$ is hopfian. Then $E$ is $J$ replaceable.

For example the above result tells us that in a finitely generated group $E$ if $E^{\prime}$ is a free product of a finite number $n, n \geqslant 2$, of nontrivial finitely generated residually finite groups then $E$ is $J$ replaceable.

COROllary 4. Let $E$ obey the minimal condition on proper prefactors and let $E$ be an HIR group. If the abelian homomorphic images of $E$ are cancelable in direct products then $E$ is cancelable in direct products.

Since abelian groups with the minimal condition on subgroups are cancelable [1], we see

COROLlary 5. Let $E$ obey the minimal condition on normal subgroups. Then $E$ is cancelable in direct products.

3.3 Proof of Theorem 3. We follow the methods of proof of Theorem 6 in [3]. Suppose the assertion is false. Then we may choose a prefactor $M$ of $E$ which is not $R$ replaceable but such that any proper prefactor of $M$ is $R$ replaceable.

Let $G=C \times M=D \times N, M \approx N$, but $C \times R \neq D \times R$. If $M \beta$ and $N \alpha$ are proper prefactors of $N$ and $M$, respectively, then from (2) of [3] we see

$$
C \times R \times R \approx D \times R \times R
$$

which implies

$$
C \times R \approx D \times R,
$$


contrary to hypothesis. Hence either $M \beta$ or $N \alpha$ is not a proper prefactor of $N$ or $M$, respectively. Suppose then that $N \alpha$ is not a proper prefactor of $M$. Hence either $N \alpha=M$ or otherwise $\alpha$ is an isomorphism on $N$. In the latter situation $N \cap C=1$. Since $N^{\prime}=\left(N^{\prime} \cap C^{\prime}\right) \times\left(N^{\prime} \cap M^{\prime}\right), N \alpha=M$ implies that $M^{\prime} \cap N^{\prime}=N^{\prime} \alpha=(N \alpha)^{\prime}=M^{\prime}$ so that $M^{\prime} \subset N^{\prime}$. Also $N \cap C=1 \mathrm{im}-$ plies $N^{\prime} \subset M^{\prime}$. Hence either $M^{\prime} \subset N^{\prime}$ or $N^{\prime} \subset M^{\prime}$.

Either one of these situations implies that $M^{\prime}=N^{\prime}$. For say $M^{\prime} \subset N^{\prime}$ so that $N^{\prime}=M^{\prime} \times\left(N^{\prime} \cap C\right)$. If $\theta$ is an isomorphism of $N$ onto $M$ then $N^{\prime} \theta=M^{\prime}$ and one can easily verify that for any positive integer $n$,

$$
M^{\prime}=M^{\prime} \theta^{n} \times\left(N^{\prime} \cap C\right) \theta \times\left(N^{\prime} \cap C\right) \theta^{2} \times \cdots \times\left(N^{\prime} \cap C\right) \theta^{n} .
$$

If $N^{\prime} \cap C \neq 1$ the above implies that $E$ is not an HIR group. Hence $N^{\prime} \cap C=1$ and $N^{\prime}=M^{\prime}$ so that

$$
G / M^{\prime} \approx\left(M / M^{\prime}\right) \times C \approx\left(N / N^{\prime}\right) \times D,
$$

which again implies $R \times C \approx R \times D$ contrary to assumption. This completes the proof.

4. Hopficity and $J$ replacement. The hopficity of a group is sometimes a useful property in studying other (often seemingly unrelated) aspects of the group. For example, Smelkin [11] shows that the unrefinable $(m)$ nilpotent decompositions of certain hopfian groups must be isomorphic. The study of cancelation in direct products and of $J$ replacement seems in many cases to be facilitated by assumptions regarding the hopficity of certain groups [3]. For example we might note Corollary 3 of Theorem 3 of this paper and Theorem A of [3] mentioned in the introduction to this paper. In the way of motivation for this section, we would, in particular, like to mention the following results of [3].

THEOREM B. Let $G=C \times M=D \times N$ with $M \approx N$ and let either $C^{\prime} \cap$ $N^{\prime}$ or $D^{\prime} \cap M^{\prime}$ be abelian. Also let $M / M^{\prime \prime}$ be $J$ replaceable. Then if $G \times J$ is hopfian, then $C \times J \approx D \times J$.

TheOREM C. Suppose $G=C \times M=D \times N, M \approx N$ and $C / C^{\prime \prime}$ and $M / M^{\prime \prime}$ are $J$ replaceable. Let $C^{\prime} \cap N^{\prime}$ or $D^{\prime} \cap M^{\prime}$ be abelian and let $M \times J$ be hopfian. Then $C \times J \approx D \times J$.

These last two results lead us to reraise the following question: If $A$ is hopfian need $A \times J$ be hopfian? Several partial results are contained in [7]. In view of the relation to this question to $J$ replacement we prove

THEOREM 4. If $A$ is a hopfian group with an infinite cyclic center, $A \times J$ is hopfian. 


\subsection{Some preliminary arguments.}

LEMMA 6. Let $y, b$ be free generators of a free abelian group $F$ of rank two. Let $\alpha$ be an automorphism of $F$ with

$$
y \alpha=y^{s} b^{j}, \quad b \alpha=y^{p} b^{t}, \quad s t-j p=1 .
$$

Then if $n$ is any positive integer we have

$$
y \alpha^{n}=y^{q_{n}} b^{e_{n}} \quad \text { with } q_{n}=s^{n} \bmod p .
$$

Proof. Set $A=\left[\begin{array}{l}s, j \\ p, t\end{array}\right]$ and note that $\bmod p, A=\left[\begin{array}{l}s, j \\ 0, t\end{array}\right]$.

LEMMA 7. Let $B$ be an arbitrary group with an infinite cyclic center $\langle b\rangle$. Let $G=\langle y\rangle \times B$ be the direct product of an infinite cyclic group with $B$. Let $\langle y\rangle \times B=\langle u\rangle \times C$ with $u \neq 1$. Let $u=y^{s} \bmod B$. Then the exponent $s$ uniquely determines $C$. That is, if we also have a second decomposition of $G$, $G=\langle y\rangle \times B=\langle v\rangle \times D$ and $v=y^{s} \bmod B, v \neq 1$, then $C \approx D$.

Proof. First observe that if $s=0$, the result is trivial. For then $u \in B$ so that if $E=\langle u\rangle, B=E \times(B \cap C)$ so that

$$
C \approx G / E \approx\langle y\rangle \times(B \cap C) \approx B .
$$

Similarly if $s=0, D \approx B$. We suppose then that $s \neq 0$. Let $Z(B)=\langle b\rangle$, $Z(C)=\langle c\rangle$. Let $u=y^{s} b^{j}, c=y^{p} b^{t}$, where without loss of generality, st $-p j$ $=1$. Now if $(\langle y\rangle \times B) / E=C_{1}$ then $C \approx C_{1}$. But note that since $\langle y\rangle \times$ $\langle b\rangle=\langle c\rangle \times E, C_{1}$ is generated by the cyclic subgroup generated by $c_{1}=$ $c E / E$ and by $B_{1}=(B E) / E \approx B$. Hence $C_{1}=\left\langle c_{1}\right\rangle B_{1}$. Note also,

$$
c_{1}^{s}=c^{s} E=y^{p s} b^{i s} E, \quad y^{s}=b^{-j} \bmod E, \quad y^{p s}=b^{-j s} \bmod E
$$

so $c_{1}^{s}=b^{-p j+s t} E=b E$. In summary,

$$
\begin{gathered}
C \approx C_{1}=\left\langle c_{1}\right\rangle B_{1}, \quad\left(c_{1}, B_{1}\right)=1, \quad\left[C_{1}: B_{1}\right]=s, \\
B_{1} \approx B, \quad\left\langle c_{1}^{s}\right\rangle=Z\left(B_{1}\right) .
\end{gathered}
$$

Similarly, it follows that we can find a group $D_{2}, D \approx D_{2}, D_{2}=\left\langle d_{2}\right\rangle B_{2}$,

$$
\left(d_{2}, B_{2}\right)=1, \quad\left[D_{2}: B_{2}\right]=s, \quad B_{2} \approx B, \quad\left\langle d_{2}^{s}\right\rangle=Z\left(B_{2}\right) .
$$

Now it is clear that $C_{1} \approx D_{2}$. In fact, if $\theta$ is an isomorphism of $B_{1}$ onto $B_{2}$ we must have $c_{1}^{s} \theta=d_{2}^{\bar{s}}$, where either $\bar{s}=s$ or $\bar{s}=-s$. In the first case we can extend $\theta$ to an isomorphism of $C_{1}$ onto $D_{2}$ by defining $c_{1} \theta=d_{2}$. If $\bar{s}=-s$ we can extend $\theta$ by defining $c_{1} \theta=d_{2}^{-1}$. It is easy to check that these extensions actually give isomorphisms.

4.2 Proof of Theorem 4. Let $G=\langle w\rangle \times A$. Let $\alpha$ be a surjective endomorphism of $G$. Then $\alpha$ induces an automorphism on the center of $G, Z(G)[7, \mathrm{p}$. 454]. Also $G=\left\langle w \alpha^{n}\right\rangle \times A \alpha^{n}$ for all $n \geqslant 0$ [7, p. 450]. These facts imply that if $y$ is a central element of $G$ with $y \alpha=w$, then $G=\langle y\rangle \times A \alpha^{-1}$.

Let $B=A \alpha^{-1}$. Let $b$ be a generator of $Z(B)$. Since $y, b$ freely generate 
$Z(G)$ we may write $y \alpha=y^{s} b^{j}, b \alpha=y^{p} b^{t}$, where without loss of generality, $s t-p j=1$.

Choose $n \geqslant 2$ so that $s^{n}=s \bmod p$. Using Lemma 6 and its notation, we see that we may find $r$ such that $s-r p=q_{n}$. Then for this $n$,

$$
\langle y\rangle \times B=\left\langle y \alpha^{n}\right\rangle \times B \alpha^{n}=\left\langle y^{q_{n}} b^{e_{n}}\right\rangle \times B \alpha^{n} .
$$

But

$$
\begin{aligned}
\langle y\rangle \times B & =\langle y \alpha\rangle \times B a=\left\langle y^{s} b^{j}\right\rangle \times B \alpha \\
& =\left\langle\left(y b^{-r}\right) \alpha\right\rangle \times B \alpha=\left\langle y^{s-r} b^{j-r t}\right\rangle \times B \alpha .
\end{aligned}
$$

Hence we may invoke Lemma 7 (with $C=B \alpha^{n}, D=B \alpha$ ) to conclude $A=B \alpha \approx B \alpha^{n}=A \alpha^{n-1}$. Hence $\alpha$ must be injective.

In conclusion we point out that a list of references dealing with hopficity may be found in [6].

\section{REFERENCES}

1. P. Crawley and B. Jonsson, Refinements for infinite direct decompositions of algebraic systems, Pacific J. Math. 14 (1964), 797-855.

2. P. Hall, Finite conditions for soluble groups, Proc. London Math. Soc. 4 (1959), $419-436$.

3. R. Hirshon, Cancellation and hopficity in direct products, J. Algebra 50 (1978), 26-33.

4. $\ldots$, The cancellation of an infinite cyclic group in direct products, Arch. Math. 26 (1975), $134-138$.

5. Some cancellation theorems with applications to nilpotent groups, J. Austral. Math. Soc. Ser. A 23 (1977), 147-165.

6. The intersection of the subgroups of finite index in some finitely presented groups, Proc. Amer. Math. Soc. 53 (1975), 32-36.

7., A conjecture on hopficity and related results, Arch. Math. (Basel) 22 (1971), $449-455$.

8. J. M. Tyrer Jones, Direct products and the Hopf property, J. Austral. Math. Soc. 27 (1974), 174-198.

9. A. G. Kurosh, The theory of groups, Vol. 2, Chelsea, New York, 1966, p. 81.

10. D. I. Moldavanski, Certain subgroups of groups with a single defining relation, Sibirsk. Mat. Ż. 8 (1966), 1370-1384.

11. A. L. Smelkin, On the isomorphism of nilpotent decompositions of torsion free nilpotent groups, Sibirsk. Mat. Ż. 4 (1963), 1412-1425. (Russian)

Department of Mathematics, Polytechnic Institute of New York, Brookiyn, New YORK 11201 